\title{
A POLAR-BASED DEMAPPER OF 8PSK DEMODULATION FOR DVB-S2 SYSTEMS
}

\author{
Anthony Barré, Emmanuel Boutillon
}

Université Européenne de Bretagne, UBS

Lab-STICC, UMR 6285

56321 Lorient, FRANCE

E-mail: emmanuel.boutillon@univ-ubs.fr

\author{
Neysser Blas, Daniel Diaz
}

INICTEL-UNI

Av. San Luis 1771, San Borja, Lima 41, Lima, Peru

E-mail:ddiaz@inictel-uni.edu.pe

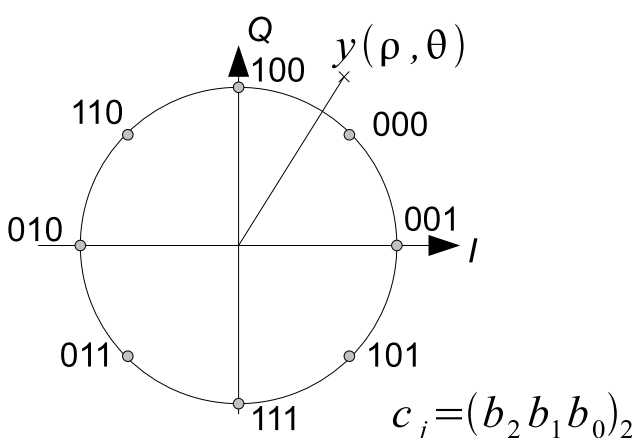

Index Terms - Receivers, Digital communication, Digital video broadcasting, Satellite communication, Telecommunications, Phase shift keying

\section{INTRODUCTION}

In DVB-S2, the demodulation is done in four successive steps: time synchronization followed by frequency synchronization, phase correction, and the generation of the Log Likelihood Ratio (LLR) of the transmitted bits [1]. In [2], the authors presented a synchronization method using polar coordinates. In this paper, we extend the use of polar coordinates to also compute the LLR. When using an 8-PSK modulation (see Fig. 1), assuming an Additive White Gaussian Noise (AWGN) channel, the exact computation of the LLR $L^{i}$, associated to the three bits $b_{i}, i=0,1,2$ of the 8 -PSK symbol is given by

$$
L^{i}(y)=\log \frac{\sum_{c \in C_{i}^{0}} e^{-\frac{(c-y)^{2}}{2 \sigma^{2}}}}{\sum_{c \in C_{i}^{1}} e^{-\frac{(c-y)^{2}}{2 \sigma^{2}}}}
$$

where $C_{i}^{0}$ (respectively $C_{i}^{1}$ ) is the subset of the points of the constellation so that the $i^{\text {th }}$ bit, $i \in\{0,1,2\}$, is equal to $b_{i}=0$ (respectively, $\left.b_{i}=1\right), y$ is the received point from the channel and $\sigma^{2}$ is the variance of the noise. This expression

\footnotetext{
${ }^{1}$ The authors thank the "Région Bretagne" and the "European Funds for Regional Development"(FEDER) for funding materials used in the study in the frame of the PALMYRE II project.
}

Fig. 1. Modulation 8-PSK and its associated binary mapping

is too complex to compute directly and could be simplified using an approximation.

In this paper, we propose a different approach by directly approximating eq. (1) in the polar domain, i.e. by using the modulus $\rho$ and the phase $\theta$ of the received point $y=\rho e^{j \theta}$ as shown in Fig. 2. Since the polar coordinate is the natural base to represent the 8-PSK modulation, the resulting demapper is much simpler than the LLR receiver based on Cartesian coordinates.

The remainder of the paper is organized as follows. Sec. 2 briefly reviews the techniques used in the state of the art to compute the LLRs. Sec. 3 explains the quantization rules for the output LLRs and the input polar coordinates. Sec. 4 describes the approximation of the ideal model by linear interpolations. Sec. 5 gives simulation results and complexity estimation. Finally, sec. 6 summerizes the paper with some conclusive remarks.

\section{STATE-OF-THE-ART}

In this section, we briefly describe the conventional LLR computation. First, the logarithm of a sum of exponentials can be simplified by considering only the most significant term inside a logarithm (max-log approximation), i.e. 


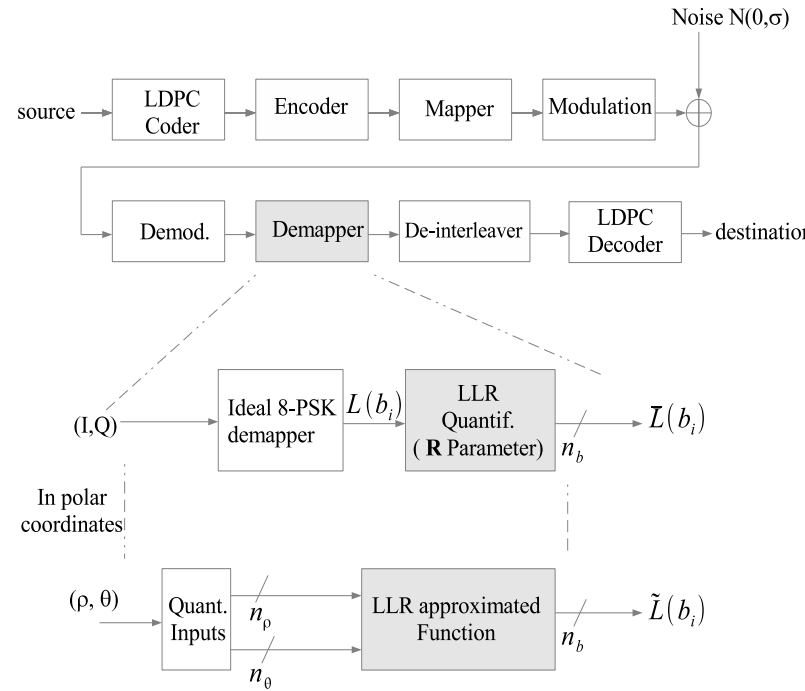

Fig. 2. Steps of DVB-S2 transmission

$$
\log \left(e^{a}+e^{b}\right) \cong \max (a, b) .
$$

Using this approximation, eq. (1) can be rewritten as

$L_{m}^{2}(y)=\max \left(D_{0}, D_{1}, D_{2}, D_{3}\right)-\max \left(D_{4}, D_{5}, D_{6}, D_{7}\right)$

$L_{m}^{1}(y)=\max \left(D_{0}, D_{1}, D_{4}, D_{5}\right)-\max \left(D_{2}, D_{3}, D_{6}, D_{7}\right)$

$L_{m}^{0}(y)=\max \left(D_{0}, D_{2}, D_{4}, D_{6}\right)-\max \left(D_{1}, D_{3}, D_{5}, D_{7}\right)$,

where $D_{j}, j=0,1, \ldots, 7$ is given by

$$
D_{j}=\log \left(e^{\frac{-\left|y-c_{j}\right|^{2}}{2 \sigma^{2}}}\right)=\frac{-\left|y-c_{j}\right|^{2}}{2 \sigma^{2}}
$$

and the index $m$ stands for "max-log approximation". The computation of $L_{m}$ can be further reduced using:

$$
L_{m}^{i}(y)=\frac{1}{2 \sigma^{2}}\left(\left|c_{i}^{1}-y\right|^{2}-\left|c_{i}^{0}-y\right|^{2}\right)
$$

where $c_{i}^{1}=\arg \min \left\{|c-y| / c \in C_{i}^{1}\right\}$ and $c_{i}^{0}=\arg \min \{\mid c-$ $\left.y \mid / c \in C_{i}^{0}\right\}$. The complexity of the computation of (4) has two components: first, (4) implies the selection of the two closest points for $i=0,1,2$. Among those 6 points, 3 are identical and correspond to the closest point of the 8-PSK constellation to the received symbol $y$, i.e., there are in fact only 4 distances. Second, (4) requires the computation of 4 quadratic distances in the complex domain, 3 subtractions and finally, 3 normalizations by the $1 / 2 \sigma^{2}$ factor. Most of the state of the art deals with the computation of (4), with methods to determine the points $c_{i}^{0}$ and $c_{i}^{1}$, for $i=0,1,2$, as described in [3], [4].

Another way to approximate $L$ is to use directly the received in-phase and in-quadrature signal [5]. This method can

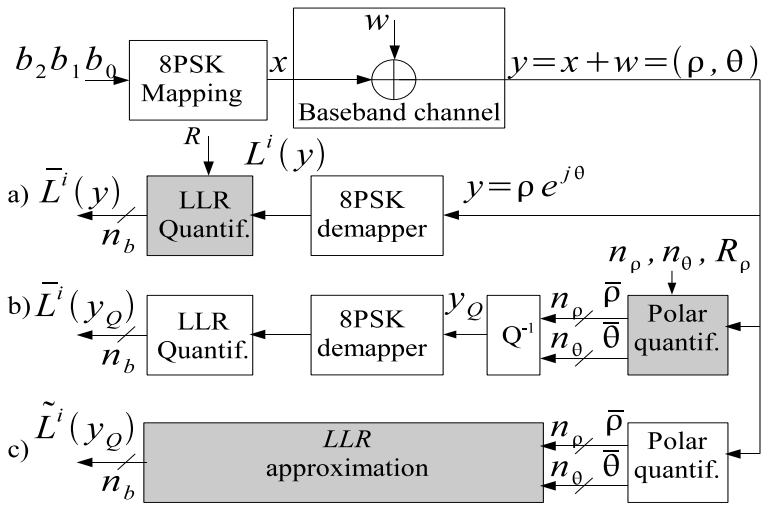

Fig. 3. The 3 steps of the quantization process

be applied when the 8-PSK constellation contains the points:

$$
c_{j}=e^{j \frac{(2 j+1) \pi}{8}}, j=0,1, \ldots, 7
$$

associated with a Gray mapping. In this case, the LLRs can be approximated using I, Q and a scaling factor given by the ratio of the signal amplitude $\rho$ multiplied by one over the noise variance $\sigma^{2}$ :

$$
\begin{aligned}
L_{g}^{0}(y) & =\frac{\rho}{\sigma^{2}} Q \\
L_{g}^{1}(y) & =\frac{\rho}{\sigma^{2}} I \\
L_{g}^{2}(y) & =\frac{\rho}{\sigma^{2}}(|I|-|Q|)
\end{aligned}
$$

This 8-PSK constellation is similar to the DBV-S2 8-PSK constellation with a rotation of $\pi / 8$. Thus, performing a rotation of $-\pi / 8$ of the received point allows to use directly this method. The computation of $L_{g}$ seems very simple, since it requires only 4 multipliers (one to compute $A \frac{\rho}{\sigma^{2}}$, plus 3 for each $L_{g}^{i}$ values). Nevertheless, the computation of $\rho=$ $\sqrt{I^{2}+Q^{2}}$ is not straightforward. Moreover, it requires also a rotation of $-\pi / 8$ in order to be applied in the context of the DVB-S2 standard. Finally, $L_{g}$ gives only a rough approximation of $L$.

\section{QUANTIZATION RULES}

In this section, we define a method to derive the fixed point model from the floating point model. Fig. 3 shows the three steps of the quantization process. The first two steps are to determine the quantization rules to obtain the LLR quantized on $n_{b}$ bits (the input of the LDPC decoder, see Fig. 3.a) and then quantize the input $\rho$ and $\theta$ (see Fig. 3.b). The third step is the linear approximation of the ideal quantized function (see Fig. 3.c). In this section, only the first two steps are described. The last step is presented in Section 4. 


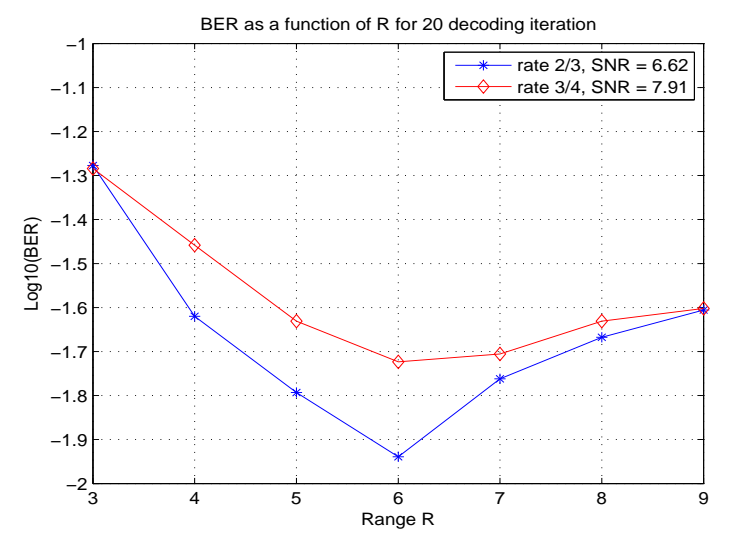

Fig. 4. Minimization of BER as a function of the quantization range $R$ for rate 2/3 and 3/4(long frame DBV-S2 LDPC code with 8-PSK modulation)

\subsection{Quantization of $L L R$ values}

The quantization law to obtain the quantized version $\bar{L}^{i}(y)$ of $L^{i}(y)$ coded on $n_{b}$ bits is given in equation (8):

$$
\bar{L}^{i}(y)=\left\lfloor\operatorname{sat}\left(L^{i}(y), R\right) \times \frac{2^{n_{b}-1}-1.5}{R}+0.5\right\rfloor
$$

where $\lfloor x\rfloor$ is the greatest integer smaller or equal than $x$ and $\operatorname{sat}(L, R)$ represents the saturation of $L$ to $\pm(R+\epsilon)$ outside the interval $[-R, R]$, where $\epsilon$ is an almost zero positive number. With this quantization scheme, if $L(y) \geq R$ then $\bar{L}(y)=$ $2^{n_{b}-1}-1$ and if $L(y) \leq-R$ then $\bar{L}(y)=-\left(2^{n_{b}-1}+1\right)$.

For each code rate, the value of $R$ is determined by simulation. Fig. 4 shows the output Bit Error Rate (BER) for energy per symbol over noise $\left(E_{s} / N_{0}\right)$ equal to $6.62 \mathrm{~dB}$ (rate 2/3 LDPC) and $7.91 \mathrm{~dB}$ (rate 3/4 LDPC) with 8-PSK modulation. The LLR are quantized on $n_{b}=5$ bits. The LDPC decoding algorithm used in the simulation is the min-sum algorithm with vertical scheduling, similar to the one presented in [7]. To reduce the time of simulation, the number of decoding iterations is limited to 20 . The curve shows that the optimal value for these two code rates is by $R=6.0$.

\subsection{Quantization of polar coordinate}

We propose to use polar coordinate to simplify the LLR computation. Thus, each received points will be first transformed in polar coordinates $y=\rho e^{j \theta}$; then $\rho \in[0,+\infty)$ and $\theta \in$ $[0,2 \pi)$ are quantized into $\bar{\rho}$ and $\bar{\theta}$ respectively on $n_{\rho}$ and $n_{\theta}$ bits according to the rules:

$$
\begin{gathered}
\bar{\rho}=\left\lfloor\operatorname{sat}\left(\rho, R_{\rho}\right) \times \frac{2^{n_{\rho}}-1.5}{R_{\rho}}+0.5\right\rfloor \\
\bar{\theta}=\left\lfloor\frac{\theta}{2 \pi} 2^{n_{\theta}}+0.5\right\rfloor \bmod 2^{n_{\theta}}
\end{gathered}
$$

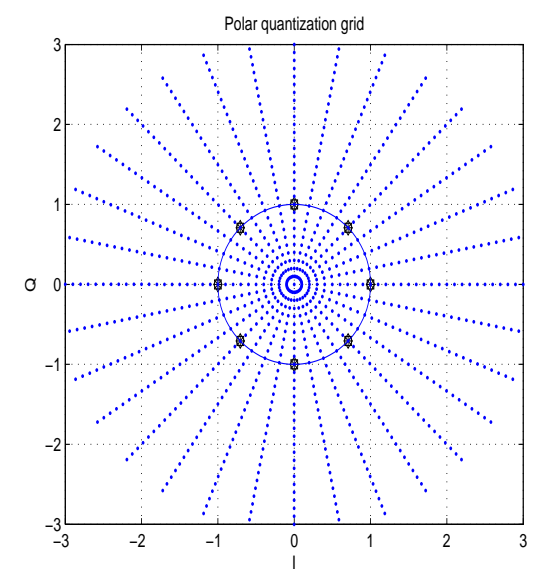

Fig. 5. Quantization in polar coordinate, $n_{\rho}=5, n_{\theta}=5$

From the integer value of $\bar{\rho}$ and $\bar{\theta}$, the quantized value $\rho_{Q}$ and $\theta_{Q}$ are given by

$$
\begin{gathered}
\rho_{Q}=\bar{\rho} \times \frac{R_{\rho}}{2^{n_{\rho}}-1} \\
\theta_{Q}=\bar{\theta} \times \frac{2 \pi}{2^{n_{\theta}}}
\end{gathered}
$$

From $\rho_{Q}$ and $\theta_{Q}$, the quantized value $y_{Q}$ of $y$ is given by

$$
y_{Q}=\rho_{Q} e^{j \theta_{Q}}
$$

In practice, we select the number of bits of $n_{\rho}$ and $n_{\theta}$ so that the absolute quantization error of $\bar{L}$ is bounded by 1 . In other words, $\forall y,\left|\bar{L}^{i}(y)-\bar{L}^{i}\left(y_{Q}\right)\right| \leq 1$. We obtained $n_{\rho}=5$ bits, $R_{\rho}=3.2$ and $n_{\theta}=7$. Figures 5 shows an example of polar quantization grid for $n_{\rho}=5, R_{\rho}=3.2$ and $n_{\theta}=5$.

The quantization rules are now fixed (see Fig. 3.b). In the next chapter, we study the approximation of the LLR (see Fig. 3.c).

\section{APPROXIMATION OF LLR COMPUTATION}

Using the quantization rules (8) with the value of $R=6.0$, we plot on Fig. 6 the quantized LLR value $\bar{L}^{i}, i=0,1,2$ for $\bar{\rho}=10\left(\rho_{Q}=1\right.$, according to (11)) as a function of $\bar{\theta}$ $\left(\theta_{Q}=2 \pi \bar{\theta} / 128 \mathrm{rd}\right.$, according to (10)). The quantized LLR has a very regular periodic triangular shape that can be easily interpolated. Fig. 7 shows the variation of $\bar{L}^{0}$ for several values of $\rho$ as a function of $\bar{\theta}$. One can note the periodic triangular shape, except for high value of $\rho$ due to the saturation process between -15 and 15 ( $n_{b}=5$ bits). We can see that the LLR can be approximated by a piecewise linear function. The shape of this function does not depend on the value of $\sigma$. To avoid the direct computation of the LLRs, we propose to approximate $L^{i}$ by a simple piecewise linear function defined as:

$$
\tilde{L}^{i}=s^{i}(\bar{\theta}) \operatorname{sat}\left(\left\lfloor\bar{\rho} \times \alpha \times h^{i}(\bar{\theta})+0.5\right\rfloor, 2^{n_{\theta}-1}-1\right)
$$




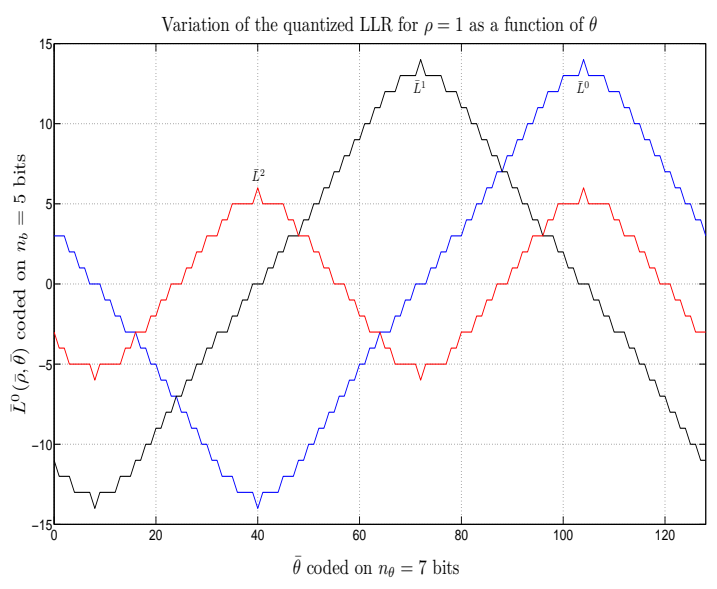

Fig. 6. Quantization of LLR for $\rho=1$ as a function of $\theta$

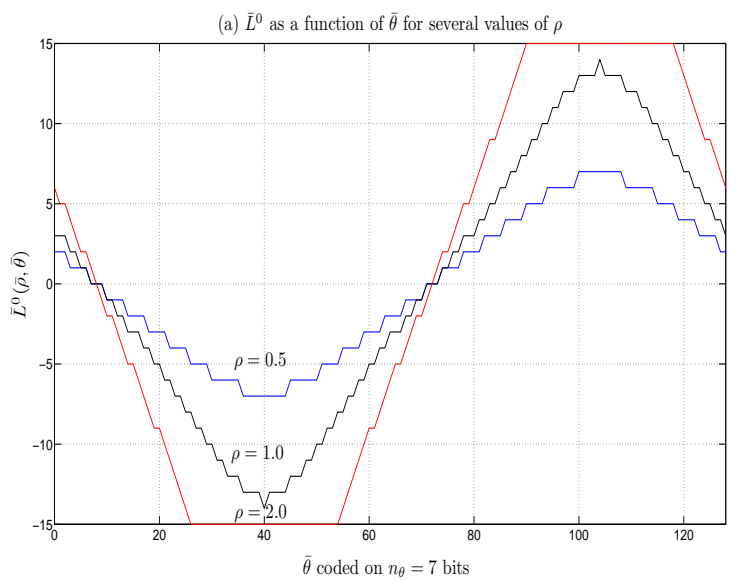

Fig. 7. Quantization of $\bar{L}^{2}$ for $\rho=0.5,1.0$ and 2.0

where $s^{i}(\bar{\theta})$ is a function that defines the sign of the $i^{t h}$ LLR, $h^{i}(\bar{\theta})$ performs translation, symmetry and saturation on $\bar{\theta}$ so that $h^{i}(\bar{\theta})$ belongs to $[0,31]$ and $\alpha$ is a scaling factor that minimizes the BER at the output of the LDPC decoder. The computation of $h^{0}(\bar{\theta})$ and $s^{0}(\bar{\theta})$ is given in Algorithm 1. For the computation of $h^{1}(\bar{\theta})$ and $s^{1}(\bar{\theta})$, step a in Algorithm 1 is replaced by $h^{1}(\bar{\theta})=\left(\bar{\theta}-2^{n_{\theta}-4}-2^{n_{\theta}-2}\right) \bmod 2^{n_{\theta}}$, steps $\mathrm{b}, \mathrm{c}$ and d remain unchanged. The computation of $h^{2}(\bar{\theta})$ and $s^{2}(\bar{\theta})$ is given in Algorithm 2 .

Fig. 8 shows the steps of the quantization process to compute $\tilde{L}^{0}$. The same architecture can be conceived to compute $\tilde{L}^{1}$ and $\tilde{L}^{2}$. The value of $\alpha$ is set to $\alpha=2^{-4}+2^{-6}$ in order to have both good performance and to reduce the product $\bar{\rho} \times \alpha$ to a simple addition of two binary shifted versions of $\bar{\rho}$. The value of $\bar{\rho} \times \alpha$ is coded on 9 bits. It is then multiplied by $h^{i}(\bar{\theta})$ coded on 5 bits to produce a result on 14 bits. The saturation and quantization unit reduced the final result from 15 bits to 5 bits. Note also that the final multiplication by $s^{i}(\bar{\theta})= \pm 1$ is a simple change of sign that doesn't require a multiplier. a) Rotation of $\bar{\theta}$ by $\pi / 8$ (symmetrical distribution) $h^{0}(\bar{\theta})=\left(\bar{\theta}-2^{n_{\theta}-4}\right) \bmod 2^{n_{\theta}}$

b) Translation of $h^{0}(\bar{\theta})$ $s^{0}(\bar{\theta})=-1$

if $h^{0}(\bar{\theta})>2^{n_{\theta}-1}$ then

$h^{0}(\bar{\theta})=h^{0}(\bar{\theta})-2^{n_{\theta}-1}$ $s^{0}(\bar{\theta})=1$

end

c) Symmetry of $h^{0}(\bar{\theta})$

if $h^{0}(\bar{\theta})>2^{n_{\theta}-2}$ then

$h^{0}(\bar{\theta})=2^{n_{\theta}-1}-h^{0}(\bar{\theta})$

end

d) Saturation $h^{0}(\bar{\theta})=\min \left(2^{n_{\theta}-2}-2, h^{0}(\bar{\theta})\right)$;

Algorithm 1: Computation of $h^{0}(\bar{\theta})$ and $s^{0}(\bar{\theta})$

a) Rotation of $\bar{\theta}$ by $\pi / 8+\pi / 4$

$h^{2}(\bar{\theta})=\left(\bar{\theta}-2^{n_{\theta}-4}-2^{n_{\theta}-3}\right) \bmod 2^{n_{\theta}-1}$

b) Translation of $h^{2}(\bar{\theta})$

$s^{2}(\bar{\theta})=1$

if $h^{2}(\bar{\theta})>2^{n_{\theta}-2}$ then

$h^{2}(\bar{\theta})=h^{2}(\bar{\theta})-2^{n_{\theta}-2}$

$s^{2}(\bar{\theta})=-1$

end

c) Symmetry of $h^{2}(\bar{\theta})$

if $h^{2}(\bar{\theta})>2^{n_{\theta}-3}$ then

$h^{2}(\bar{\theta})=2^{n_{\theta}-2}-h^{2}(\bar{\theta})$

end

d) Saturation $h^{2}(\bar{\theta})=\min \left(2^{n_{\theta}-3}-2, h^{2}(\bar{\theta})\right)$;

Algorithm 2: Computation of $h^{2}(\bar{\theta})$ and $s^{2}(\bar{\theta})$

\section{SIMULATION RESULTS}

Fig. 9 shows the BER as a function of the SNR for a $2 / 3$ and a 3/4 LDPC code rate (code size equals to $N=64800$ bits). We can see that the performance of our proposed method is quite similar to the optimal one. Table 1 shows in the first line of data the Mean Square Error (MSE) between exact and approximated LLR computation of the 3 methods. The remaining lines of the table compare the complexity of the 3 methods..

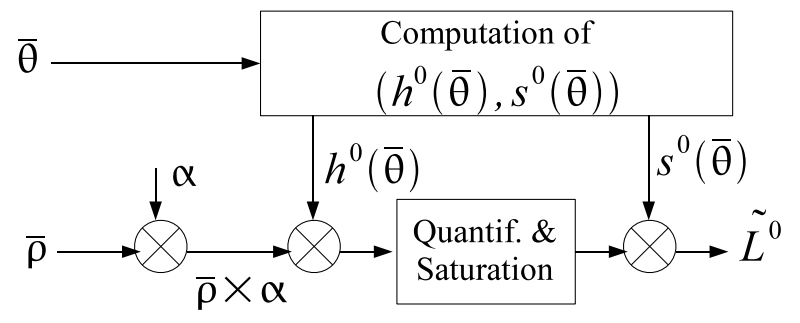

Fig. 8. Architecture of computation of $\tilde{L}^{0}$ 


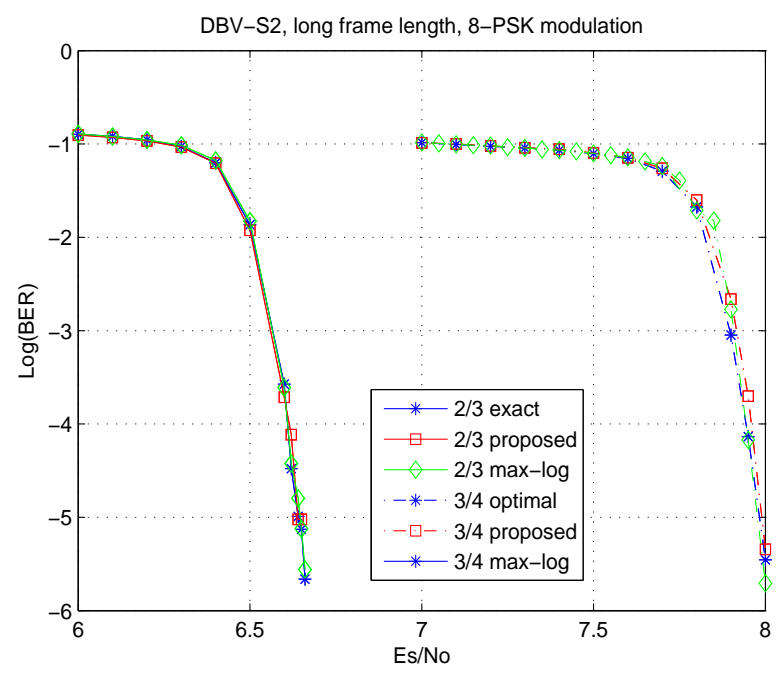

Fig. 9. Binary Error Rate as a function of the signal to noise ratio $E_{s} / N_{0}$ for LPDC rate $2 / 3$ and $3 / 4$

Table 1. Comparaison of methods of LLR generation

\begin{tabular}{|c|c|c|c|}
\hline Method & Max [4] & $I$ and $Q[5]$ & Polar \\
\hline MSE & 0.94 & 1.54 & 0.89 \\
\hline Sel. closest points & yes & no & no \\
\hline Square Distance & 4 & 0 & 0 \\
\hline$\sqrt{I^{2}+Q^{2}}$ & 0 & 1 & 0 \\
\hline$-\pi / 8$ rotation & 0 & 1 & 0 \\
\hline Add/sub & 3 & 1 & 1 \\
\hline Product & 3 & 4 & 3 \\
\hline$\left(h^{i}(\bar{\theta}), s^{i}(\bar{\theta})\right)_{i=1,2,3}$ & 0 & 0 & 1 \\
\hline
\end{tabular}

We can notice that the polar approximation is slightly more accurate than the max-log method [4] and, overall, is simpler to implement. In terms of performance, both max$\log$ approximation and polar approximatoin lead to similar results. The main application domain of the polar LLR computation is for "polar based" demodulator [2], since it avoid the use of a CORDIC to transform back polar coordinates to cartesian coordinnates.

\section{CONCLUSION}

This paper proposes a novel approach for the demapping of 8-PSK modulation. The idea is to use polar coordinates instead of Cartesian coordinates. The polar domain allows approximating the LLR using simple linear interpolation. This kind of function is very simple to compute and allows us to obtain a better reliability and lower complexity than the stateof-the-art methods. This approach is particularly well suited for demodulator that uses polar-based coordinates to perform some of the synchronization tasks, since it avoids a polar to
Cartesian transformation.

The method can be extended to the 16-APSK or the 32APSK modulations, since the plot of LLR as a function of $\bar{\theta}$ gives also triangular shapes. In other words, the method shown in the paper can serve as a basis for implementing all PSK modulations in the DVB-S2 standard.

\section{REFERENCES}

[1] ETSI EN 302 307, v. 1.1.2 Digital Video Broadcasting (DVB); Second generation framing structure, channel coding and modulation systems for broadcasting, interactive services, news gathering and other broadband satellite applications, June 2006.

[2] E. Boutillon; P. Kim; C. Roland; D.-G. Oh, Efficient Multiplierless Architecture for Frame Synchronization in DVB-S2 Standard, SISP 2011, Beirut, Oct. 2011.

[3] Jianing Su; Zhenghao Lu; Xiaopeng Yu; Changhui Hu, A Novel Low Complexity Soft-decision Demapper for QPSK 8-PSK Demodulation of DVB-S2 system, International Conference of Electron Devices and Solid-State Circuits (EDSSC'11), pp. 17-18 Tianjin (China), Nov. 2011.

[4] Jang Woong Park; Myung Hoon Sunwoo; Pan Soo Kim; Dae-Ig Chang, Low Complexity Soft-Decision Demapper for High Order Modulation of DVB-S2 system International SoC Design Conference, ISOCC'08, vol. 02, pp. 37-40, Nov. 2008.

[5] Michael K. Cheng; Dariush Divsalar; Stephanie Duy, Structured Low-Density Parity-Check Codes with Bandwidth Efficient Modulation, Proc. SPIE 7349, Wireless Sensing and Processing IV, Orlando, April, 2009.

[6] J. E. Volder, "The CORDIC Trigonometric Computing Technique", IRE Transactions on Electronic Computers, Sept. 1959.

[7] M. Li, C. Abdel Nour, C. Jgo, J. Yang and C. Douillard, A Shuffled Iterative Bit-interleaved Coded Modulation Receiver for the DVB-T2 Standard: Design, Implementation and FPGA Prototyping, 2011 IEEE Workshop on Signal Processing Systems (SiPS), pp. 55 60, Beirut (Lebanon), Oct. 2011. 